\title{
Letter
}

\section{Rotating black hole and quintessence}

\author{
Sushant G. Ghosh ${ }^{1,2, a}$ \\ ${ }^{1}$ Centre for Theoretical Physics, Jamia Millia Islamia, New Delhi 110025, India \\ 2 Astrophysics and Cosmology Research Unit, School of Mathematics, Statistics and Computer Science, University of KwaZulu-Natal, \\ Private Bag 54001, Durban 4000, South Africa
}

Received: 23 December 2015 / Accepted: 30 March 2016 / Published online: 22 April 2016

(c) The Author(s) 2016. This article is published with open access at Springerlink.com

\begin{abstract}
We discuss spherically symmetric exact solutions of the Einstein equations for quintessential matter surrounding a black hole, which has an additional parameter $(\omega)$ due to the quintessential matter, apart from the mass $(M)$. In turn, we employ the Newman-Janis complex transformation to this spherical quintessence black hole solution and present a rotating counterpart that is identified, for $\alpha=-e^{2} \neq 0$ and $\omega=1 / 3$, exactly as the Kerr-Newman black hole, and as the Kerr black hole when $\alpha=0$. Interestingly, for a given value of parameter $\omega$, there exists a critical rotation parameter ( $a=a_{E}$ ), which corresponds to an extremal black hole with degenerate horizons, while for $a<a_{E}$, it describes a nonextremal black hole with Cauchy and event horizons, and no black hole for $a>a_{E}$. We find that the extremal value $a_{E}$ is also influenced by the parameter $\omega$ and so is the ergoregion.

The accelerating expansion of the Universe implies the crucial contribution of matter with negative pressure to the evolution of the Universe, which could be the cosmological constant or so-called quintessence matter. If quintessence matter exists all over in the Universe, it can also be around a black hole. In this letter, we are interested in getting the rotating counterpart of the solution to the Einstein equations obtained with the assumption of spherical symmetry, with the quintessence matter as a source of energy-momentum obtained by Kislev [1] and was also rigorously analyzed by himself and others [1-8]. Let us commence with the general
\end{abstract} spherically symmetric spacetime

$\mathrm{d} s^{2}=g_{a b} \otimes \mathrm{d} x^{a} \otimes \mathrm{d} x^{b} \quad(a, b=0,1,2,3)$,

with $g_{a b}=\operatorname{diag}\left(-f(r), f(r)^{-1}, r^{2}, r^{2} \sin ^{2} \theta\right)$, and the energy-momentum tensor for the quintessence [1] is given by

$$
\begin{aligned}
T_{t}^{t} & =T_{r}^{r}=\rho_{q}, \\
T_{\theta}^{\theta} & =T_{\phi}^{\phi}=-\frac{1}{2} \rho_{q}(3 \omega+1) .
\end{aligned}
$$

a e-mail: sgghosh@gmail.com; sghosh2@jmi.ac.in
On using the Einstein equations $G_{a b}=T_{a b}$, one obtains

$f(r)=1-\frac{r_{g}}{r}+\frac{\beta}{r^{3 \omega+1}}$,

where $\beta$ and $r_{g}$ are the normalization factors. The density of quintessence matter $\rho_{q}$ is given by

$\rho_{q}=\frac{\beta}{2} \frac{3 \omega}{r^{3(\omega+1)}}$.

The sign of the normalization constant should coincide with the sign of the matter state parameter, i.e. $\beta \omega \geq 0$, implying that $\beta$ is negative for the quintessence and hence we choose $\alpha=-\beta$. Thus the metric of exact spherically symmetric solutions for the Einstein equations describing black holes surrounded by quintessential matter with the energymomentum tensor (2) is given by

$$
\begin{aligned}
\mathrm{d} s^{2}= & {\left[1-\frac{2 M}{r}-\frac{\alpha}{r^{3 \omega+1}}\right] \mathrm{d} t^{2} } \\
& -\frac{\mathrm{d} r^{2}}{\left[1-\frac{2 M}{r}-\frac{\alpha}{r^{3 \omega+1}}\right]}-r^{2} \mathrm{~d} \Omega^{2} .
\end{aligned}
$$

Here $r_{g}$ is related to the black hole mass via $r_{g}=2 M$, and $\omega$ is the quintessential state parameter. The Ricci scalar for the solution reads

$R=R_{a b}^{a b}=\frac{9 \alpha^{2} \omega^{2}\left(9 \omega^{2}+6 \omega+3\right)}{2 r^{2(3 \omega+1)}}$,

indicating scalar polynomial singularity at $r=0$ if $\omega \neq$ $\left\{0, \frac{1}{3},-1\right\}$. Thus we have a general form of exact spherically symmetric solutions for the Einstein equations describing black holes surrounded by quintessential matter. The parameter $\omega$ has to have the range $-1<\omega<-1 / 3$ for a de Sitter horizon, and this causes the acceleration, and $-1 / 3<\omega<0$ for the asymptotically flat solution. It is the most general spherically symmetric static solution of Einstein's field equation coupled with quintessence matter as a source. For $\alpha=0$, it reduces to the Schwarzschild solution. The case with the 
relativistic matter state parameter $\omega=1 / 3$, with $\alpha=-e^{2}$, corresponds to a Reissner-Nordstrom black hole with

$f(r)=1-\frac{r_{g}}{r}+\frac{e^{2}}{r^{2}}$.

The solution for the Reissner-Nordstrom black hole surrounded by the quintessence gives

$f(r)=1-\frac{r_{g}}{r}+\frac{e^{2}}{r^{2}}-\frac{\alpha}{r^{3 \omega+1}}$.

The borderline case of $\omega=-1$ of the extraordinary quintessence covers the cosmological constant term, and the spacetime (5) reduces to the Schwarzschild-de Sitter black hole.

The purpose of this letter is to seek the generalization of the solution (5) to the axially symmetric case or to the Kerrlike metric. The Kerr metric [9] is beyond question the most extraordinary exact solution in the Einstein theory of general relativity, which represents the prototypic black hole that can arise from gravitational collapse, which contains an event horizon [10]. It is well known that Kerr black holes enjoy many interesting properties distinct from its non-spinning counterpart, i.e., from the Schwarzschild black hole. However, there is a surprising connection between the two black holes of Einstein theory, and it was analyzed by Newman and Janis [11-14] that the Kerr metric [9] could be obtained from the Schwarzschild metric using a complex transformation within the framework of the Newman-Penrose formalism [15]. A similar procedure was applied to the ReissnerNordstrm metric to generate the previously unknown KerrNewman metric [12]. It is an ingenious algorithm to construct a metric for rotating black hole from static spherically symmetric solutions in Einstein gravity. The NewmanJanis method has proved to be prosperous in generating new stationary solutions of the Einstein field equations and the method has also been studied outside the domain of general relativity [17-26], although it may lead to additional stresses $[20,21,26,27]$. For possible physical interpretations of the algorithm, see [28,29], and for discussions on more general complex transformations, see $[16,28,29]$. For a review of the Newman-Janis algorithm see, e.g., [30].

Next, we wish to derive a rotating analogue of the static spherically symmetric quintessence solution (5) by employing the Newman-Janis [11] complex transformation. To attempt similarly for the static quintessence solution (5) to generate a rotating counterpart, we take the quintessence solution (5) and perform the Eddington-Finkelstein coordinate transformation,

$\mathrm{d} u=\mathrm{d} t-\left[1-\frac{2 M}{r}-\frac{\alpha}{r^{3 \omega+1}}\right] \mathrm{d} r$, so that the metric takes the form

$\mathrm{d} s^{2}=\left[1-\frac{2 M}{r}-\frac{\alpha}{r^{3 \omega+1}}\right] \mathrm{d} u^{2}-2 \mathrm{~d} u \mathrm{~d} r-r^{2} \mathrm{~d} \Omega^{2}$.

Following the Newman-Janis prescription [11,25], we can write the metric in terms of the null tetrad, $Z^{a}=\left(l^{a}, n^{a}\right.$, $\left.m^{a}, \bar{m}^{a}\right)$, as

$g^{a b}=l^{a} n^{b}+l^{b} n^{a}-m^{a} \bar{m}^{b}-\bar{m}^{a} m^{b}$,

where the null tetrads are

$$
\begin{aligned}
l^{a} & =\delta_{r}^{a}, \\
n^{a} & =\delta_{u}^{a}-\frac{1}{2}\left[1-\frac{2 M}{r}-\frac{\alpha}{r^{3 \omega+1}}\right] \delta_{r}^{a}, \\
m^{a} & =\frac{1}{\sqrt{2} r}\left(\delta_{\theta}^{a}+\frac{i}{\sin \theta} \delta_{\phi}^{a}\right) .
\end{aligned}
$$

The null tetrads are orthonormal and obey the metric conditions

$l_{a} l^{a}=n_{a} n^{a}=(m)_{a}(m)^{a}=(\bar{m})_{a}(\bar{m})^{a}=0$,

$l_{a}(m)^{a}=l_{a}(\bar{m})^{a}=n_{a}(m)^{a}=n_{a}(\bar{m})^{a}=0$,

$l_{a} n^{a}=1, \quad(m)_{a}(\bar{m})^{a}=1$.

Now we allow for some $r$ factor in the null vectors to take on complex values. We rewrite the null vectors in the form $[25,26]$

$$
\begin{aligned}
l^{a} & =\delta_{r}^{a}, \\
n^{a} & =\left[\delta_{u}^{a}-\frac{1}{2}\left[1-M\left(\frac{1}{r}+\frac{1}{\bar{r}}\right)-\frac{\alpha}{(r \bar{r})^{\frac{3 \omega+1}{2}}}\right] \delta_{r}^{a}\right], \\
m^{a} & =\frac{1}{\sqrt{2} \bar{r}}\left(\delta_{\theta}^{a}+\frac{i}{\sin \theta} \delta_{\phi}^{a}\right),
\end{aligned}
$$

with $\bar{r}$ being the complex conjugate of $r$. Following the Newman-Janis prescription [11], we now write

$$
\begin{aligned}
x^{\prime a} & =x^{a}+i a\left(\delta_{r}^{a}-\delta_{u}^{a}\right) \cos \theta \\
& \rightarrow\left\{\begin{array}{l}
u^{\prime}=u-i a \cos \theta, \\
r^{\prime}=r+i a \cos \theta, \\
\theta^{\prime}=\theta, \\
\phi^{\prime}=\phi,
\end{array}\right.
\end{aligned}
$$

where $a$ is the rotation parameter. Simultaneously, let the null tetrad vectors $Z^{a}$ undergo a transformation $Z^{a}=$ $Z^{\prime a} \partial x^{\prime a} / \partial x^{b}$ in the usual way; we obtain

$$
\begin{aligned}
l^{a}= & \delta_{r}^{a}, \\
n^{a}= & {\left[\delta_{u}^{a}-\frac{1}{2}\left[1-\frac{2 M r}{\Sigma}-\frac{\alpha}{\Sigma^{\frac{3 \omega+1}{2}}}\right] \delta_{r}^{a}\right], } \\
m^{a}= & \frac{1}{\sqrt{2}(r+i a \cos \theta)} \\
& \times\left(i a\left(\delta_{u}^{a}-\delta_{r}^{a}\right) \sin \theta+\delta_{\theta}^{a}+\frac{i}{\sin \theta} \delta_{\phi}^{a}\right),
\end{aligned}
$$


where $\rho=r^{2}+a^{2} \cos \theta$ and we have dropped the primes. Using the tetrad (14), the non-zero component of the inverse of the new metric can be written as

$g^{u u}=-\frac{a^{2} \sin ^{2}(\theta)}{\Sigma(r, \theta)}, \quad g^{u \phi}=-\frac{a}{\Sigma(r, \theta)}$,

$g^{\phi \phi}=-\frac{1}{\Sigma(r, \theta) \sin ^{2} \theta}, \quad g^{\theta \theta}=-\frac{1}{\Sigma(r, \theta)}$,

$g^{r r}=-\frac{a^{2} \sin ^{2} \theta}{\Sigma(r, \theta)}-\zeta(r, \theta), \quad g^{r \phi}=\frac{a}{\Sigma(r, \theta)}$,

$g^{u r}=\frac{a^{2} \sin ^{2}(\theta)}{\Sigma(r, \theta)}+1$,

where

$\zeta(r, \theta)=1-\frac{2 M r}{\Sigma}-\frac{\alpha}{\Sigma^{\frac{3 \omega+1}{2}}}$.

From the new null tetrad, a new metric is constructed using (10), which takes the form

$$
\begin{aligned}
\mathrm{d} s^{2}= & \zeta(r, \theta) \mathrm{d} u^{2}+2 \mathrm{~d} u \mathrm{~d} r-2 a \sin ^{2} \theta \mathrm{d} r \mathrm{~d} \phi \\
& -\Sigma(r, \theta) \mathrm{d} \theta^{2}-\left[a^{2}(\zeta(r, \theta)-2)-\Sigma(r, \theta)\right] \\
& \times \sin ^{2} \theta \mathrm{d} \phi^{2}-2 a(1-\zeta(r, \theta)) \sin \theta^{2} \mathrm{~d} u \mathrm{~d} \phi,
\end{aligned}
$$

with $\Sigma(r, \theta)=r^{2}+a^{2} \cos ^{2} \theta$.

Thus we have obtained rotating analogue of the static black hole metric (5)

$$
\begin{aligned}
\mathrm{d} s^{2}= & \zeta(r, \theta) \mathrm{d} t^{2}+\frac{\Sigma(r, \theta)}{\Delta(r)} \mathrm{d} r^{2} \\
& +2(1-\zeta(r, \theta)) \sin ^{2} \theta \mathrm{d} t \mathrm{~d} \phi-\Sigma(r, \theta) \mathrm{d} \theta^{2} \\
& -\sin ^{2} \theta\left[a^{2}(2-\zeta(r, \theta)) \sin ^{2} \theta+\Sigma(r, \theta)\right] \mathrm{d} \phi^{2} .
\end{aligned}
$$

In order to simplify the notation we introduce the following quantities:

$$
\begin{aligned}
\Delta(r) & =\zeta(r, \theta) \Sigma(r, \theta)+a^{2} \sin ^{2} \theta \\
& =r^{2}+a^{2}-2 M r-\frac{\alpha}{\Sigma^{\frac{3 \omega-1}{2}}},
\end{aligned}
$$

inside the metric and we write down the line element explicitly in Boyer-Lindquist coordinates defined by the coordinate transformation

$\mathrm{d} u=\mathrm{d} t-\left(\frac{r^{2}+a^{2}}{\Delta}\right) \mathrm{d} r, \quad \mathrm{~d} \phi=\mathrm{d} \phi^{\prime}-\frac{a}{\Delta} \mathrm{d} r$.

In the above and henceforth, we omit writing the dependence on $\theta$ and $r$ in the function $\Delta$ as well as in $\Sigma$. Then this metric could be cast in the more familiar Boyer-Lindquist coordinates to read

$$
\begin{aligned}
\mathrm{d} s^{2}= & \frac{\Delta-a^{2} \sin ^{2} \theta}{\Sigma} \mathrm{d} t^{2}-\frac{\Sigma}{\Delta} \mathrm{d} r^{2} \\
& +2 a \sin ^{2} \theta\left(1-\frac{\Delta-a^{2} \sin ^{2} \theta}{\Sigma}\right) \mathrm{d} t \mathrm{~d} \phi-\Sigma \mathrm{d} \theta^{2}
\end{aligned}
$$

$$
-\sin ^{2} \theta\left[\Sigma+a^{2} \sin ^{2} \theta\left(2-\frac{\Delta-a^{2} \sin ^{2} \theta}{\Sigma}\right)\right] \mathrm{d} \phi^{2},
$$

with $\Delta$ and $\Sigma$ as defined above. This is a rotating black hole metric which for $\alpha=0$ reduces to the Kerr black hole, while in the particular case $a=0$, it reconstructs the Schwarzschild solution surrounded by the quintessence, and for definiteness, we call the metric (19) a rotating quintessence black hole which is stationary and axisymmetric with Killing vectors.

However, like the Kerr metric, the rotating quintessence black hole metric (19) is also singular at $r=0$. The metric (19) generically must have two horizons, viz., the Cauchy horizon and the event horizon. The surface of no return is known as the event horizon. The zeros of $\Delta=0$ give the horizons of the black hole, i.e., the roots of

$\Delta=r^{2}+a^{2}-2 M r-\frac{\alpha}{\Sigma^{\frac{3 \omega-1}{2}}}=0$.

This depends on $a, \alpha, \omega$, and $\theta$, and it is different from the Kerr black hole where it is $\theta$ independent. The numerical analysis of Eq. (20) suggests the possibility of two roots for a set of values of parameters, which corresponds to the two horizons of a rotating quintessence black hole metric (19). The larger and smaller roots of Eq. (20) correspond, respectively, to the event and Cauchy horizons. An extremal black hole occurs when $\Delta=0$ has a double root, i.e., when the two horizons coincide. When $\Delta=0$ has no root, i.e., no horizon exists, this means there is no black hole (cf. Fig. 1). We have explicitly shown that, for each $\omega$, one gets two horizons for $a<a_{E}$, and when $a=a_{E}$ the two horizons coincide, i.e., we have an extremal black hole with degenerate horizons (Fig. 1; Table 1). Further, for $a<a_{E}$, Eq. (20) admits two positive roots which are $\omega$ dependent (Fig. 1; Tables 2 and $3)$.

In the case $\alpha=0$, when the Kerr black hole solution is recovered, there is an event horizon with spherical topology, which is the largest root of the equation $\Delta=0$, given by

$r^{ \pm}=M \pm \sqrt{M^{2}-a^{2}}$,

for $a \leq M$. Beyond this critical value of the spin there is no event horizon and causality violations are present in the whole spacetime, with the appearance of a naked singularity. The case $\alpha=-e^{2} \neq 0$, with $\omega=1 / 3$, leads to $\Delta=$ $r^{2}+a^{2}-2 M r+e^{2}$ and the roots

$r^{ \pm}=M \pm \sqrt{M^{2}-a^{2}-e^{2}}$,

correspond to the outer and inner horizons of the KerrNewman black hole.

In general, as envisaged the black hole horizon is spherical and it is given by $\Delta=0$, which has two positive roots giving the usual outer and inner horizon and no negative roots. The numerical analysis of the algebraic equation $\Delta=0$ reveals 

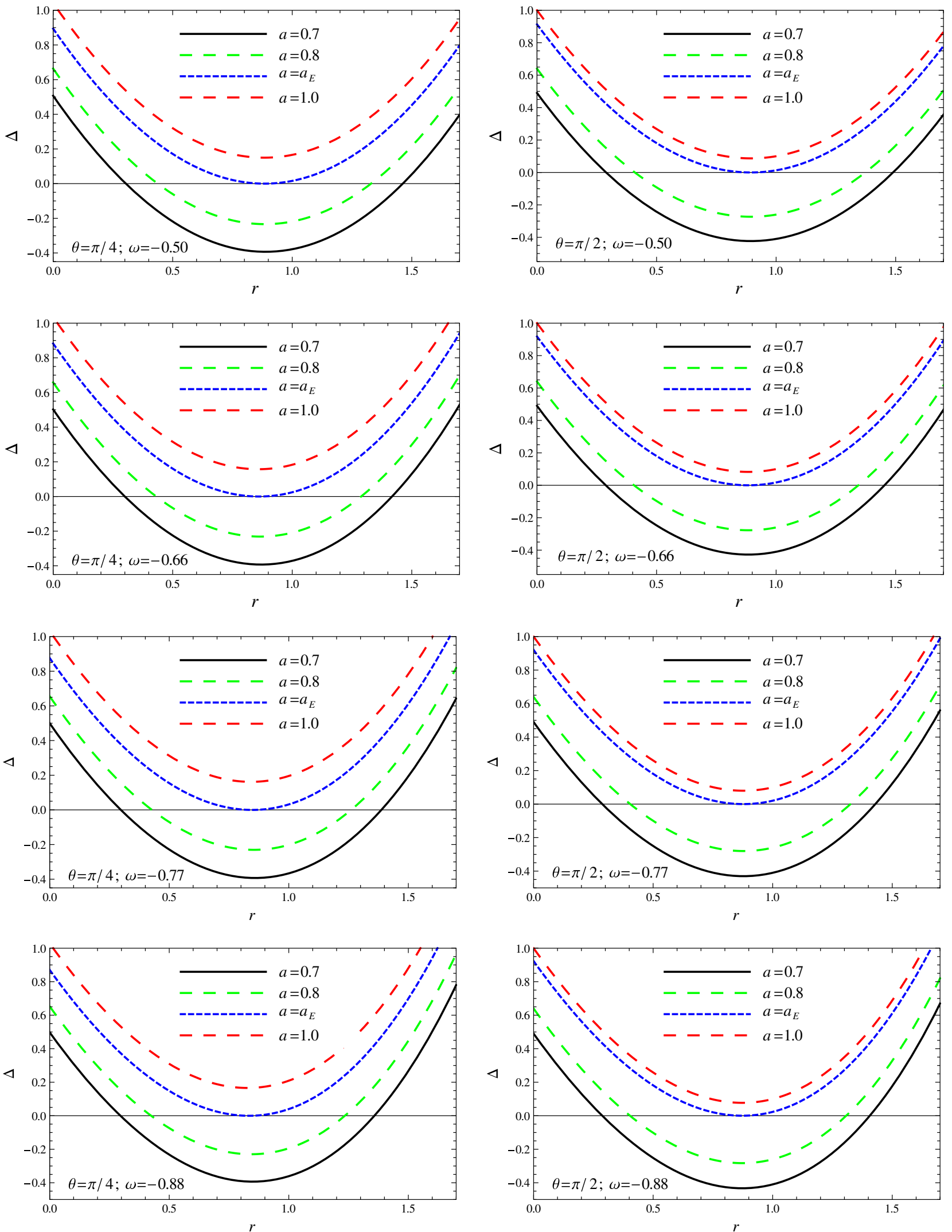

Fig. 1 Plot showing the behavior of $\Delta$ vs. $r$ for fixed values of $\alpha=-0.1$, and $M=1$ by varying $a$. The case $a=a_{E}$ corresponds to an extremal black hole 
Table 1 The effect of quintessence parameter $\omega$ on the extremal rotation parameter $\left(a_{E}\right)$ and extremal horizon $\left(r^{E}\right)$

\begin{tabular}{llllll}
\hline$\omega$ & $\theta=\pi / 4$ & & $\theta=\pi / 2$ & $r^{E}$ \\
\cline { 2 - 3 } & $a=a_{E}$ & $r^{E}$ & & $a_{E}$ & 0.894288 \\
\hline-0.50 & 0.9270821091720760 & 0.884066 & & 0.9556124089886700 & 0.883037 \\
-0.66 & 0.9243197545134466 & 0.860520 & & 0.9578436527014000 & 0.877225 \\
-0.77 & 0.9228228129512000 & 0.845995 & & 0.9593257246846765 & 0.872548 \\
-0.88 & 0.9215809590176600 & 0.832531 & & 0.9607794407624000 & 0.85 \\
\hline
\end{tabular}

Table 2 The Cauchy and event horizons of the black hole for different values of $\omega$ and $a(\theta=\pi / 4)$

\begin{tabular}{|c|c|c|c|c|c|c|c|c|}
\hline \multirow{2}{*}{$\overline{a<a_{E}}$} & \multicolumn{2}{|c|}{$\omega=-0.50$} & \multicolumn{2}{|c|}{$\omega=-0.66$} & \multicolumn{2}{|c|}{$\omega=-0.77$} & \multicolumn{2}{|c|}{$\omega=-0.88$} \\
\hline & $r^{-}$ & $r^{+}$ & $\overline{r^{-}}$ & $r^{+}$ & $\overline{r^{-}}$ & $r^{+}$ & $\overline{r^{-}}$ & $r^{+}$ \\
\hline 0.7 & 0.304099 & 1.46241 & 0.299547 & 1.41561 & 0.297165 & 1.38497 & 0.295200 & 1.35543 \\
\hline 0.8 & 0.437164 & 1.32997 & 0.430741 & 1.28681 & 0.427068 & 1.25913 & 0.423826 & 1.23282 \\
\hline 0.9 & 0.671981 & 1.09592 & 0.665427 & 1.05488 & 0.660976 & 1.02987 & 0.656497 & 1.00698 \\
\hline
\end{tabular}

Table 3 The Cauchy and event horizons of the black hole for different values of $\omega$ and $a(\theta=\pi / 2)$

\begin{tabular}{|c|c|c|c|c|c|c|c|c|}
\hline \multirow[t]{2}{*}{$a<a_{E}$} & \multicolumn{2}{|c|}{$\omega=-0.50$} & \multicolumn{2}{|c|}{$\omega=-0.66$} & \multicolumn{2}{|c|}{$\omega=-0.77$} & \multicolumn{2}{|c|}{$\omega=-0.88$} \\
\hline & $r^{-}$ & $r^{+}$ & $r^{-}$ & $r^{+}$ & $r^{-}$ & $r^{+}$ & $r^{-}$ & $r^{+}$ \\
\hline 0.7 & 0.289008 & 1.48923 & 0.287523 & 1.45172 & 0.286949 & 1.42732 & 0.286574 & 1.40387 \\
\hline 0.8 & 0.408981 & 1.37298 & 0.405586 & 1.34310 & 0.404079 & 1.32413 & 0.402983 & 1.30616 \\
\hline 0.9 & 0.596924 & 1.18918 & 0.588102 & 1.17125 & 0.583599 & 1.16050 & 0.579960 & 1.15067 \\
\hline
\end{tabular}

that it is possible to find non-vanishing values of the parameters $a, \omega$ and $\alpha$ for which $\Delta$ has a minimum, and that $\Delta=0$ admits two positive roots $r^{ \pm}$(cf. Fig. 1).

The static limit or ergo surface is given by $g_{t t}=0$, i.e.,

$\left(r^{2}+a^{2} \cos ^{2} \theta\right)-2 M r-\frac{\alpha}{\Sigma^{\frac{3 \omega-1}{2}}}=0$.

The behavior of the static limit surface is shown in Fig. 2. The two surfaces, viz. the event horizon and the static limit surface, meet at the poles and the region between them gives the ergoregion admitting negative energy orbits, i.e., the region between $r_{+}^{E H}<r<r_{+}^{S L S}$ is called the ergoregion, where the asymptotic time translation Killing field $\xi^{a}=\left(\frac{\partial}{\partial t}\right)^{a}$ becomes spacelike and an observer follows the orbit of $\xi^{a}$. It turns out that the shape of the ergoregion, therefore, depends on the spin $a$ and the parameter $\omega$. Interestingly, the quintessence matter does influence the shape of the ergoregion as described in Fig. 3 when compared with the analogous situation of the Kerr black hole. Indeed, we have demonstrated that the ergoregion is vulnerable to the parameter $\omega$ and the ergoregion becomes more prolate; the ergoregion area increases as the value of the parameter $\omega$ increases. Further, we find that for a given value of $\omega$, one can find a critical parameter $a^{C}$ such that the horizons are disconnected for $a>a^{C}$ (cf. Fig. 3).
Penrose [31] surprised everyone when he suggested that energy can be extracted from a rotating black hole. The Penrose process [31] relies on the presence of an ergoregion, which for the solution (19) grows with the increase of the parameter $\omega$ as well with spin $a$ (cf. Fig. 3). Thus the parameter $\omega$ is likely to have an impact on the energy extraction. It will also be useful to further study the geometrical properties, causal structures, and thermodynamics of this black hole solution. All these and related issues are being investigated.

In this letter, we have used the complex transformations pointed out by Newman and Janis [11], to obtain rotating solutions from the static counterparts for the quintessential matter surrounding a black hole. Interestingly, the limit as $a \rightarrow 0$ is still correct from the point of view of the obtained solution, but it is easy to see that the metric obtained by the complex transformation is likely to generate additional stress [20,26,27]. It may be pointed out that in the general relativity case the source, if it exists, is the same for both a black hole and its rotating counterpart (obtained by Newman-Janis complex transformations), e.g., the vacuum for both Schwarzschild and Kerr black holes, and the charge for the Reissner-Nordstrom and Kerr-Newman black holes. The source for the solution (5) is just quintessence matter, whereas its rotating counterpart (19), in addition to quintessence matter, has some additional stresses. 

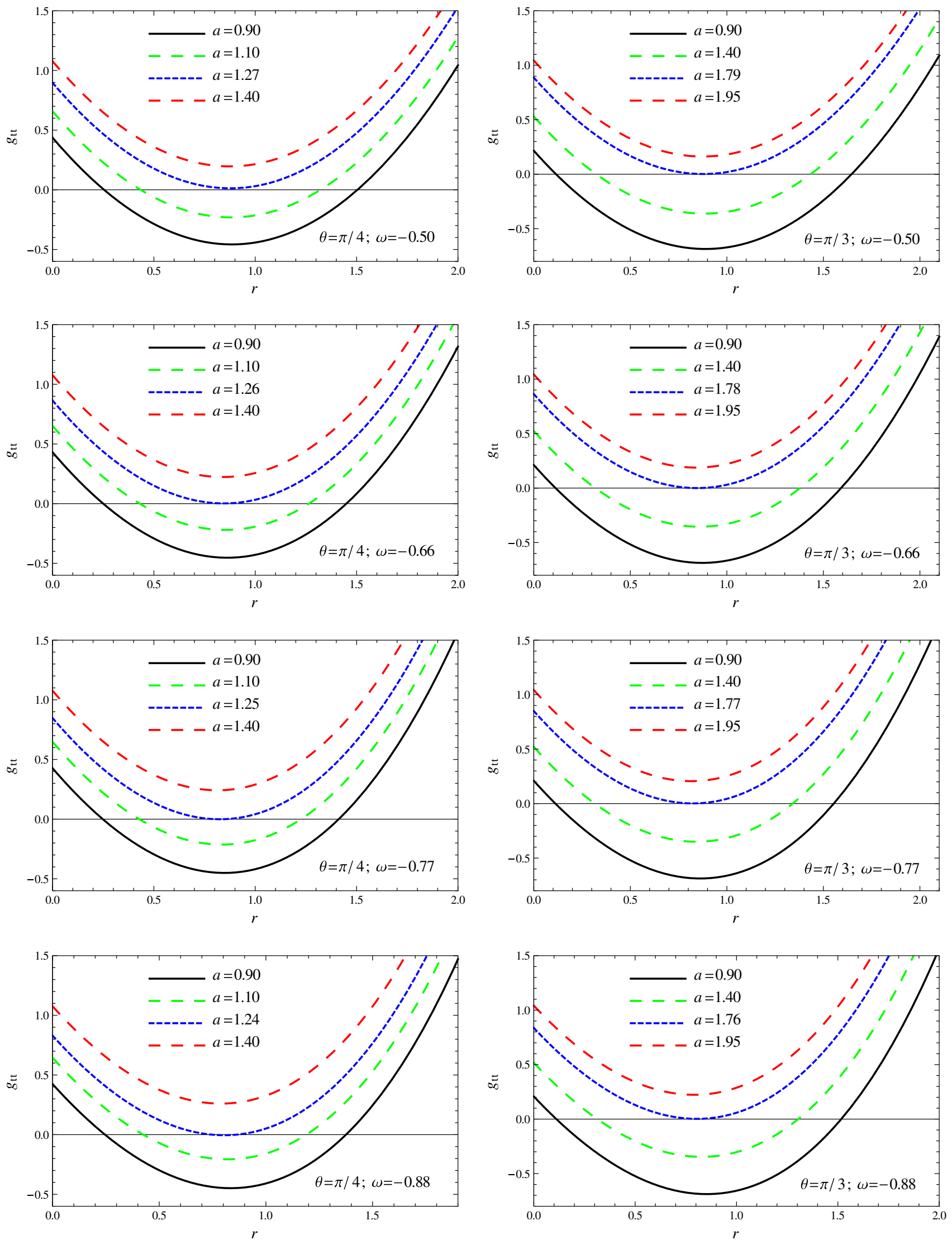

Fig. 2 Plot showing the behavior of $g_{t t}$ vs. $r$ for fixed values of $\alpha=-1$ and $M=1$ by varying $a$ 

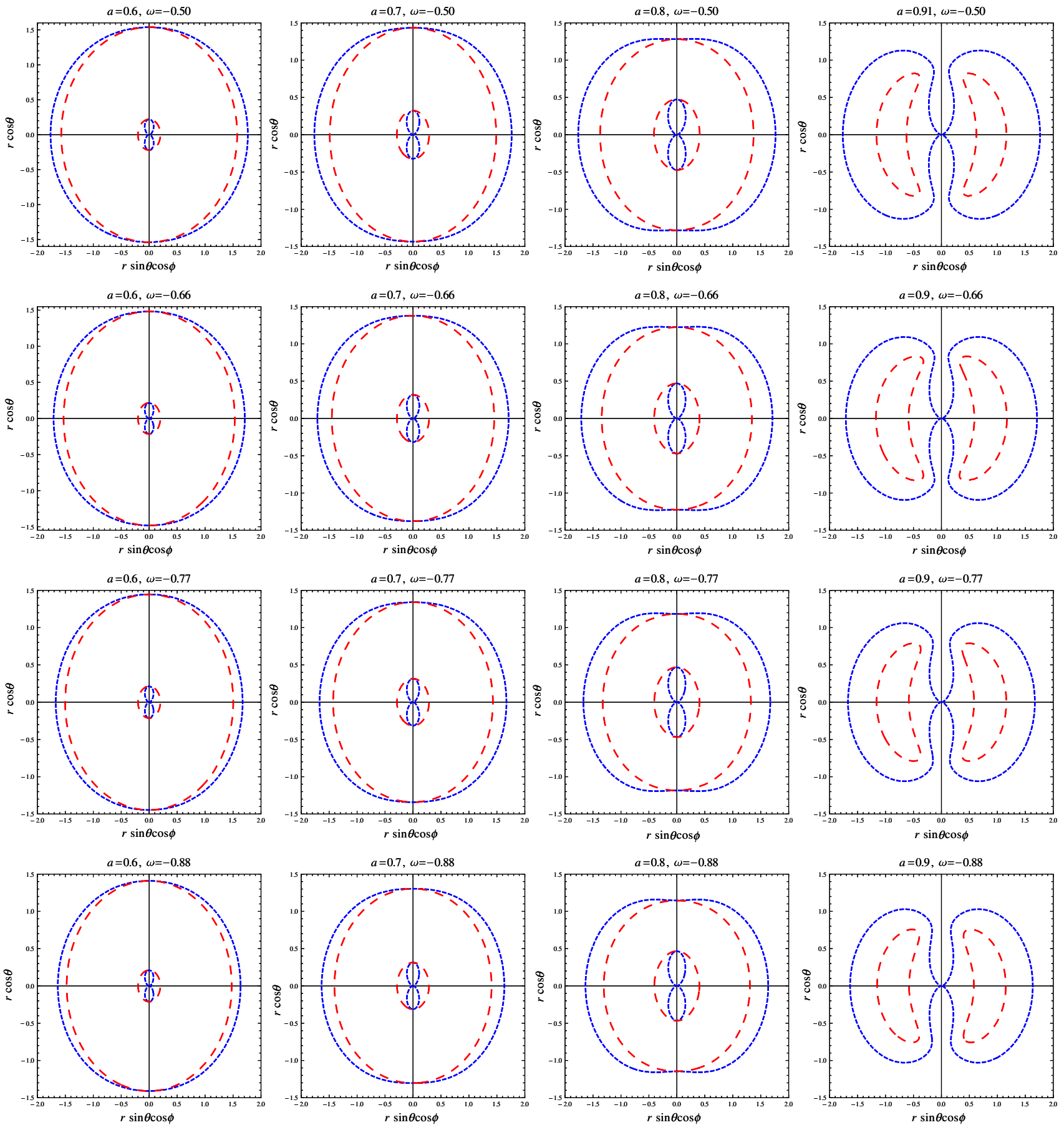

Fig. 3 Plot showing the variation of the shape of ergoregion in the $x z$ plane with parameter $\omega$, for different values of $a$, of the rotating black hole. The blue and the red lines correspond, respectively, to static limit

Acknowledgments We would like to thank SERB-DST Research Project Grant No. SB/S2/HEP-008/2014, and M. Amir for help in the plots. We also thank IUCAA for hospitality while this work was being done and ICTP for Grant No. OEA-NET-76.

Added in proof After this work was completed, we learned of a similar work by Toshmatov et al. [32], which appeared in the arXiv a couple of days before.

surfaces and horizons. The outer blue line corresponds to the static limit surface, whereas the two red lines correspond to the two horizons

Open Access This article is distributed under the terms of the Creative Commons Attribution 4.0 International License (http://creativecomm ons.org/licenses/by/4.0/), which permits unrestricted use, distribution, and reproduction in any medium, provided you give appropriate credit to the original author(s) and the source, provide a link to the Creative Commons license, and indicate if changes were made. Funded by SCOAP ${ }^{3}$. 


\section{References}

1. V.V. Kiselev, Class. Quantum Gravity 20, 1187 (2003)

2. Sb Chen, Jl Jing, Class. Quantum Gravity 22, 4651 (2005)

3. Y. Zhang, Y.X. Gui, Class. Quantum Gravity 23, 6141 (2006)

4. S. Chen, B. Wang, R. Su, Phys. Rev. D 77, 124011 (2008)

5. Y.H. Wei, Z.H. Chu, Chin. Phys. Lett. 28, 100403 (2011)

6. B.B. Thomas, M. Saleh, T.C. Kofane, Gen. Relativ. Gravit. 44, 2181 (2012)

7. S. Fernando, Mod. Phys. Lett. A 28, 1350189 (2013)

8. R. Tharanath, V.C. Kuriakose, Mod. Phys. Lett. A 28, 1350003 (2013)

9. R.P. Kerr, Phys. Rev. Lett. D 11, 237 (1963)

10. B. Carter, Black Holes (Gordon and Breach, New York, 1973)

11. E.T. Newman, A.I. Janis, J. Math. Phys. 6, 915 (1965)

12. E.T. Newman, E. Couch, K. Chinnapared, A. Exton, A. Prakash, R. Torrence, J. Math. Phys. 6, 918 (1965)

13. E.T. Newman, J. Math. Phys. 14, 774 (1973)

14. E.T. Newman, The Remarkable Efficacy of Complex Methods in General Relativity, ed. by B.R. Iyer, et al. Highlights in Gravitation and Cosmology. Proceedings of the International Conference on Gravitation and Cosmology (Goa, 1987), (Cambridge University Press, Cambridge, 1988) p. 67

15. E. Newman, R. Penrose, J. Math. Phys. 3, 566 (1962)
16. S.P. Drake, P. Szekeres, Gen. Relativ. Gravit. 32, 445 (2000)

17. C. Bambi, L. Modesto, Phys. Lett. B 721, 329 (2013)

18. B. Toshmatov, B. Ahmedov, A. Abdujabbarov, Z. Stuchlik, Phys. Rev. D 89(10), 104017 (2014)

19. A. Larranaga, A. Cardenas-Avendano, D.A. Torres, Phys. Lett. B 743, $492(2015)$

20. S.G. Ghosh, S.D. Maharaj, Eur. Phys. J. C 75(1), 7 (2015)

21. J.C.S. Neves, A. Saa, Phys. Lett. B 734, 44 (2014)

22. M. Azreg-Ainou, Phys. Lett. B 730, 95 (2014)

23. S.G. Ghosh, Eur. Phys. J. C 75(11), 532 (2015)

24. S.G. Ghosh, U. Papnoi, Eur. Phys. J. C 74(8), 3016 (2014)

25. S. Capozziello, M. De laurentis, A. Stabile, Class. Quantum Gravity 27, 165008 (2010)

26. D.J. Cirilo Lombardo, Class. Quantum Gravity 21, 1407 (2004)

27. M. Carmeli, M. Kaye, Ann. Phys. 103, 97 (1977)

28. E.J. Flaherty, Hermitian and Khlerian Geometry in Relativity Lecture Notes in Physics (Springer, Berlin, 1976)

29. E.J. Flaherty, Complex Variables in Relativity, ed. by A. Held. General Relativity and Gravitation, vol. 2 (Plenum, New York, 1980) p. 207

30. R. dInverno, Introducing Einsteins Relativity (Clarendon, Oxford, 1992)

31. R. Penrose, Riv. Nuovo Cimento 1, 252 (1969)

32. B. Toshmatov, Z. Stuchlk, B. Ahmedov. arXiv:1512.01498 [gr-qc] 